\title{
Cholinergic Activity Enhances Hippocampal Long-Term Potentiation in CA1 during Walking in Rats
}

\author{
L. Stan Leung, ${ }^{1,2,5}$ Bixia Shen, ${ }^{1,2}$ Nagalingam Rajakumar, ${ }^{3,4,5}$ and Jingyi Ma $^{1,2}$ \\ Departments of ${ }^{1}$ Physiology and Pharmacology, ${ }^{2}$ Clinical Neurological Sciences, ${ }^{3}$ Psychiatry, and ${ }^{4}$ Anatomy and Cell Biology, and ${ }^{5}$ Neuroscience Program, \\ The University of Western Ontario, London, Ontario, Canada N6A 5A5
}

Long-term potentiation (LTP) at the basal-dendritic synapses of CA1 pyramidal cells was induced by a single $200 \mathrm{~Hz}$ stimulation train (0.5-1 sec duration) in freely behaving rats during one of four behavioral states: awake-immobility (IMM), walking, slow-wave sleep (SWS), and rapid eye movement sleep (REM). Field EPSPs generated by basal-dendritic excitation of CA1 were recorded before and up to $1 \mathrm{~d}$ after the tetanus. After a tetanus during any behavioral state, basal-dendritic LTP was $>170 \%$ of the baseline for the first hour after the tetanus and decayed to $\sim 120 \% 1 \mathrm{~d}$ after. LTP induced during walking was significantly larger than that induced during IMM, SWS, or REM, which had similar LTP magnitudes. To test the hypothesis that septohippocampal cholinergic activity enhanced LTP during walking as compared with IMM, rats were either pretreated with muscarinic cholinergic antagonist scopolamine or injected with IgG192saporin in the medial septum to selectively lesion cholinergic septohippocampal neurons. Pretreatment with scopolamine decreased the LTP induced during walking but did not affect that induced during IMM, such that the difference between the LTP induced during walking and IMM was abolished after scopolamine. Rats injected with IgG192-saporin showed no difference in the LTP induced during walking and IMM, and scopolamine did not reduce the LTP during walking. In contrast, sham-lesion rats showed larger LTP induced during walking than IMM, and the LTP induced during walking was attenuated by scopolamine. This is the first demonstration of an enhancement of hippocampal LTP by physiologically activated cholinergic inputs.

Key words: long-term potentiation; CA1; basal dendrites; acetylcholine; theta rhythm; spatial learning

\section{Introduction}

Long-term potentiation (LTP), a long-lasting enhancement of synaptic transmission, was first reported in anesthetized (Bliss and Lomo, 1973) and behaving animals (Bliss and GardnerMedwin, 1973). LTP is believed to be the type of synaptic plasticity that underlies learning and memory (Bliss and Collingridge, 1993; Morris and Frey, 1997).

The physiological condition that provides the optimal conditions for the induction or maintenance of LTP has not been fully explored. One approach is to study the LTP induced during different behavioral states. Previous studies using this approach focused on the medial perforant path synapses in the dentate gyrus (DG) of the hippocampus. Jones Leonard et al. (1987) showed that LTP in the DG was suppressed during slow-wave sleep (SWS) as compared with during waking. Bramham and Srebo (1989) reported that LTP in the DG occurred with a lower success rate when induced during SWS than during still-alert (awake) and rapid eye movement (REM) sleep. To our knowledge, no other central synapse has been investigated in relation to its propensity for LTP during different behavioral states.

\footnotetext{
Received July 11, 2003; revised Aug. 22, 2003; accepted Aug. 27, 2003.

This research was supported by the Canadian Institutes of Health Research MT-15685 and the Natural Sciences and Engineering Council of Canada.

Correspondence should be addressed to Dr. L. Stan Leung, Department Clinical Neurological Sciences, The University of Western Ontario, University Campus, London Health Sciences Center, London, Ontario, Canada N6A 5A5 E-mail: sleung@uwo.ca.

Copyright $\odot 2003$ Society for Neuroscience $\quad$ 0270-6474/03/239297-08\$15.00/0
}

Sleep states, including both SWS and REM, are actively generated by brainstem mechanisms (Jouvet, 1967). It has been increasingly recognized that information processing in the brain may occur during sleep states, in particular for memory consolidation (Buzsaki, 1989; Smith, 1996). In the hippocampus, Pavlides and Winson (1989) showed that the place firing of a hippocampal neuron became more robust after the rat had bouts of SWS in the place field of the neuron. Interactions among hippocampal neurons, acquired during active exploration, were maintained during SWS and REM (Wilson and McNaughton, 1994; Louie and Wilson, 2001).

Central cholinergic activity facilitates human and animal learning (Deutsch, 1991; Decker and McGaugh, 1991). Of particular significance is that a decrease in cholinergic function is partly responsible for the decline in cognitive functions in Alzheimer's patients (Gallagher and Nicolle, 1993; Kasa et al., 1997; Sirvio, 1999; Bartus, 2000). There is experimental evidence that cholinergic activity facilitates LTP in various areas of the brain (Hasselmo and Barkai, 1995; Hasselmo, 1999; Boyd et al., 2000). The facilitation of hippocampal LTP by acetylcholine has been shown mainly by pharmacological and other nonphysiological, experimental manipulations. Application of cholinergic agonists enhanced hippocampal LTP in vitro (Blitzer et al., 1990; Auerbach and Segal, 1996), and stimulation of the medial septal cholinergic pathway enhanced hippocampal LTP in vivo (Galey et al., 1994; Markevich et al., 1997). However, enhancement of hippocampal LTP by physiological release of acetylcholine has not been shown. 
In this study, we investigated the LTP of the basal-dendritic excitatory synapses in CA1 of behaving rats by applying brief tetanic stimulations during different behavioral states. Release of acetylcholine in the hippocampus varies as a function of behavioral states, and it is higher during walking than during IMM (Dudar et al., 1979). Thus, we hypothesize that LTP in CA1 is enhanced during behavioral states with high acetylcholine release, namely walking and REM, as compared with IMM and SWS. In addition, using muscarinic cholinergic blockade and selective lesion of the septohippocampal cholinergic neurons, we directly tested the hypothesis that the stronger LTP during walking than IMM was mediated by cholinergic activity in the hippocampus. The basal-dendritic synapse in CA1 is particularly appropriate for this study because of its robust LTP (Leung et al., 1992; Kaibara and Leung, 1993; Leung and Shen, 1995).

\section{Materials and Methods}

Under sodium pentobarbital anesthesia, male (250-400 gm) hooded rats were implanted bilaterally with $125 \mu \mathrm{m}$ wire electrodes in the dorsal hippocampus. The electrodes were Tefloninsulated except at the cut tips and were used for either stimulation or recording. Recording electrodes were implanted in CA1 (P3 to 4.5 according to atlas by Paxinos and Watson, 1986), straddling the CA1 cell layer on either side (Leung and Shen, 1995). The surface electrode was typically at a depth from the white matter of the neocortex to CA1 stratum oriens, and the deep (ventral) electrode was in CA1 stratum radiatum. Stimulating electrodes were placed in stratum oriens of CA1 (at about P2 to P3.5) on the same side anterior to the recording electrodes, or about homotopic on the opposite side (Leung and Shen, 1995). Two screws in the skull over the frontal cortex and cerebellum served, respectively, as the stimulus anode and the recording ground. The depth of the stimulus and recording electrodes were optimized during implantation using established electrophysiological criteria. In essence, stimulation of the stratum oriens evoked a basal-dendritic field EPSP (fEPSP) that was negative at stratum oriens and positive at the CA1 cell layer and apical dendrites (Fig. 1) (Leung and Shen, 1995). One week after surgery, the rat was habituated to the recording environment for 1-2 d. Recording was performed between 9:00 A.M. and 4:00 P.M. Photoisolated current stimulus pulses (0.2 msec duration and 10-200 $\mu \mathrm{A}$ intensity) were delivered cathodally to one stimulating electrode, using a screw in the skull as the anode. Two to three channels of evoked responses were filtered at $0.1 \mathrm{~Hz}$ to $3 \mathrm{kHz}$, sampled at $7 \mathrm{kHz}$, and averaged evoked potentials (AEPs) were acquired on-line by a custom microcomputer program. Electrode sites were subsequently confirmed by $40 \mu \mathrm{m}$ coronal brain sections stained by thionin.

A high-frequency train of $200 \mathrm{~Hz}$ of $0.5-1 \mathrm{sec}$ duration was used to induce basal-dendritic LTP in behaving rats (Leung and Shen, 1995). The $0.5-1 \mathrm{sec}$ duration of the tetanus was long enough to span several cycles of the theta rhythm (or any EEG rhythm of $<2 \mathrm{~Hz}$ ), such that stimulation was delivered to all phases of the theta rhythm, and theta phase-

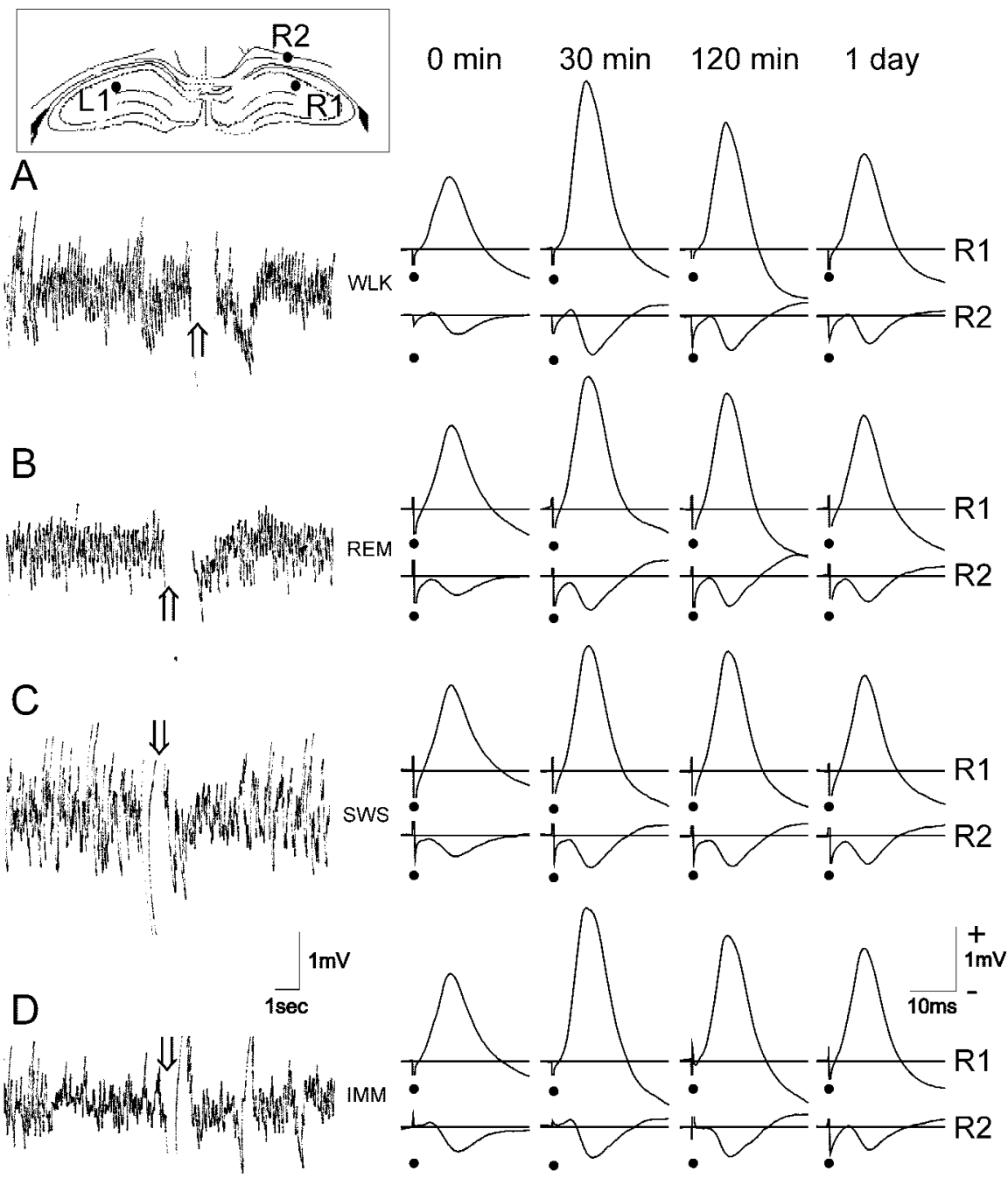

Figure 1. Basal-dendritic fEPSPs reveal larger LTP when a high-frequency train was delivered during walking compared with delivered to the basal-dendritic afferents during a given behavioral state. The behavioral state remained undisrupted by the the largest LTP compared with during REM ( $B)$, SWS ( $C$, and IMM (D). Both surface and deep electrodes show similar results. The experiments were done in the sequence shown $(A-D)$ on the same rat (913), with at least $6 \mathrm{~d}$ between experiments; the same test pulse intensity and the same tetanus intensity were used across experiments.

dependent LTP (Pavlides et al., 1988; Holscher et al., 1997) would not occur. A single tetanus train was preferred over repeated trains because a particular behavioral state (e.g., one bout of rapid eye movement sleep) may be relatively brief in duration $(<2 \mathrm{~min})$. Tetanic stimulations in CA1 were given at $1.1-1.5 \times$ the single-pulse response threshold. Four behavioral states (Vanderwolf, 1988; Leung, 1998) were distinguished: (1) awake-immobility (IMM), when the rat held its head up against gravity, with eyes open and no gross body movements, and the hippocampal EEG showed a large-amplitude irregular activity; (2) walking, when the rat was walking on a horizontal surface, and hippocampal EEG showed a regular theta rhythm; (3) slow-wave sleep (SWS), when the rat was in a sleep posture with head supported, and with high-amplitude slow waves in the neocortex and hippocampus; and (4) rapid eye movement sleep (REM), when the rat was in a sleep posture, with desynchronized neocortical EEG and regular hippocampal theta rhythm. EEGs were recorded on a polygraph during the tetanus to identify the behav- 
ioral state (Fig. 1A). Hippocampal theta frequency was estimated from the polygraph records, particularly for the time ( $1 \mathrm{sec})$ immediately preceding the tetanus. For a tetanus delivered during sleep, the animal must remain in the same type of sleep for at least $10 \mathrm{sec}$ after the tetanus. All experiments in which the tetanus induced an afterdischarge (Leung and Shen, 1995) were excluded.

All AEPs (average of eight sweeps), unless otherwise noted, were recorded during IMM. Baseline AEPs were recorded, with test pulses of $1.5-2 \times$ threshold intensity, for at least $1.5 \mathrm{hr}$ before the tetanus and at "fixed" times of 5, 10, 20, 30, 60, 90, 120, 150, $180 \mathrm{~min}$ and $1 \mathrm{~d}$ (22 hr) after the tetanus. Previous data indicated that the magnitude of LTP (when expressed as a ratio of the baseline) was not significantly different among test pulses of 1.5-2× threshold intensity (Leung and Shen, 1995). AEPs were recorded during various sleep and wake states, and the degree of LTP was not found to depend on the behavioral state during the recording. Input-output curves consisting of fEPSP responses to four or five different stimulus intensities (1-4X threshold) were recorded for the baseline and at $1 \mathrm{hr}, 2 \mathrm{hr}$, and $1 \mathrm{~d}(22 \mathrm{hr})$ after the tetanus. The slope (over 1-3 msec interval) of the fEPSP was measured from the AEPs. A grand average of all the slope measures recorded before the tetanus was used as the baseline value (100\%). The ratio of the SEM to the mean (SEM/mean) of the baseline fEPSPs ranged from 0.01-0.08, and experiments with a SEM/mean ratio $>0.08$ were discarded. For each experiment, the response after tetanus was normalized by the baseline average. The normalized responses at fixed times after the tetanus were subjected to a two-factor (group $\times$ time) repeated measure ANOVA followed by post hoc Neuman-Keuls test. Unless otherwise noted, the data reported were derived from recordings at the stratum radiatum electrode, because the fEPSPs at the latter electrode were of higher amplitude and better stability than those at the (alvear) surface electrode (Leung and Shen, 1995, 1999). The main reason is that the curvature of the hippocampus accentuates the deep (ventral) dipole field and makes its voltage gradient more gradual. However, similar magnitudes of LTP were found at both the deep and surface electrodes (Leung and Shen, 1999), and the main results of this study were confirmed at the surface electrode as well (Fig. 1). The use of positive and not negative potentials of the CA1 basal-dendritic fEPSP is similar to the recording of positive potentials in the hilus of the DG for studying LTP of the perforant-path synapses, which has been exclusively performed in behaving rats (Jones Leonard et al., 1987; Bramham and Srebro, 1989; Barnes et al., 1994; Saucier and Cain, 1995).

The same rat was subjected to repeated experiments, with the tetanus delivered at the same intensity during a different behavioral state in each experiment. Experiments were separated by $>4 \mathrm{~d}$ (typically $6-8 \mathrm{~d}$ ), and the behavioral states were selected in a quasi-random, counterbalanced time order. The response of the rat must be stable across all experiments; the criterion of stability was that the fEPSP slope during baseline (before tetanus) must be $<30 \%$ between experiments. Previous experiments showed that the basal-dendritic LTP averaged $\sim 10 \%$ at 1 week after tetanus (Leung and Shen, 1995). Repeated measures from the same rat were preferred because, for technical reasons, the variation of LTP magnitude among rats was larger than that among behavioral states. Only 7 of 14 rats completed the sequence of four behavioral states, for two main reasons: (1) the tetanus was sufficiently strong to wake the rat up from SWS or REM, or to evoke an afterdischarge in one behavioral state or (2) the baseline fEPSP changed by $>30 \%$ over the four experiments, possibly because of movement of the stimulating or recording electrodes. Subsequent experiments were conducted by inducing LTP during two behavioral states. The pair of experiments was done on the same rat within 3 weeks, in a random order, using the same stimulus intensity for test pulses and the same tetanus intensity. Again, the stability criterion was that the baseline fEPSP slope was within 30\% among experiments.

In some experiments, muscarinic cholinergic antagonist scopolamine hydrochloride $(5 \mathrm{mg} / \mathrm{kg}$ ) or an equal volume of saline was injected intraperitoneally, 15 min before tetanus. Scopolamine-saline injections were done in separate experiments within 2 weeks in the same rat, in a random order, and with the tetanus given in a fixed behavioral state (of either walking or IMM).

Under pentobarbital anesthesia, a 30 gauge cannula was placed sequentially into para-midline locations, at the left and right medial sep- tum (lateral \pm 0.5 ; anterior 0 ; ventral 7.5 ), and injections were made at a rate of $0.5 \mu \mathrm{l} / \mathrm{min}$ by means of an infusion pump through a 30 gauge Hamilton syringe. The lesion rats received IgG192-saporin (SAP) (Chemicon, Temecula, CA) at $0.14 \mu \mathrm{g} / 0.4 \mu \mathrm{l}$ per side, and the shamlesion rats received $0.4 \mu \mathrm{l}$ saline injections. The injection needle remained in place for $10 \mathrm{~min}$ before retraction to allow diffusion of the solution. At the end of experiments, saporin- and sham-lesion rats were perfused through the heart with $100 \mathrm{ml}$ of saline followed by $400 \mathrm{ml}$ of $4 \%$ paraformaldehyde fixative solution (Sigma, St. Louis, MO) in $0.1 \mathrm{M}$ phosphate buffer (PB), pH 7.4. The rat brain was removed and postfixed with $18 \%$ sucrose in PBS for $24 \mathrm{hr}$ at $4^{\circ} \mathrm{C}$. For immunocytochemistry of choline acetyltransferase (ChAT) and parvalbumin (Parv), sections were incubated first in $10 \%$ normal goat serum in $0.1 \mathrm{M}$ PBS containing $0.01 \%$ Triton X-100 (Sigma) for $1 \mathrm{hr}$ at room temperature to block nonspecific labeling. The sections were rinsed briefly in PBS and incubated at $4^{\circ} \mathrm{C}$ for $48 \mathrm{hr}$ in primary antibody solution containing mouse monoclonal ChAT (1:200; Biogenesis, Sandown, NH) and 1\% normal goat serum. Sections were washed in three changes of PBS and followed by biotin-conjugated goat anti-mouse secondary antiserum (1:200; BioCan) for $1 \mathrm{hr}$ in room temperature. The sections were then washed several times in PBS solution. While the sections were being washed, the $\mathrm{ABC}$ complex was prepared by adding equal volumes of solutions A and B mixed with PBS (1:1:100) 20 min before use (standard ABC reagent; Vector Laboratories, Burlingame, CA). The sections were incubated in the ABC solution for 1 $\mathrm{hr}$ at room temperature. After three more washes in PBS, the sections were incubated in a fumehood in a solution containing $0.05 \%$ diaminobenzidine tetrahydrochloride (DAB; Sigma) and $0.003 \%$ hydrogen peroxide in PBS at room temperature until they reached the desired color intensity (1-3 $\mathrm{min})$. The sections were then washed several times in PBS and mounted on chrome-alum gelatin-coated slides. They were dehydrated in a series of 70,95, and 100\% ethyl alcohol twice (1 min each), cleared in xylene (two times for $5 \mathrm{~min}$ ), and coverslipped with Entellan (BDH Chemicals, Poole, UK) mounting medium. Other sections of the brain were stained for acetylcholinesterase (AchE) using Timm's stain (Leung, 1987). For comparison between sham-lesion and SAP-lesion rats, the slides of each rat were processed at the same time on the same tray, using identical staining parameters.

The sites of cannulas and the electrodes were verified histologically in $40 \mu \mathrm{m}$ frozen sections of the brain stained with thionin. The number of ChAT- or Parv-positive cells were counted in a standard coronal section $(\sim 0.1 \mathrm{~mm}$ posterior to bregma) through the medial septum and diagonal band of Broca region, after a digital image was acquired at $100 \times$ magnification by a Nikon camera through a Nikon microscope.

\section{Results \\ Basal-dendritic LTP in CA1 induced different behavioral states in normal rats}

As reported before (Kaibara and Leung, 1993; Leung and Shen, 1995), basal-dendritic fEPSP evoked by ipsilateral or contralateral CA1 stimulation was negative at the basal-dendritic layer (Fig. 1A, electrode R2) and positive at the apical dendrites (Fig. $1 A$, electrode $\mathrm{R} 1$ ). The dipole field was attributed to EPSCs at the basal dendrites after stimulation of the CA3 afferents (Leung et al., 1992; Kaibara and Leung, 1993). For 14 rats used for the study of state-dependent LTP, the single-pulse threshold of the basaldendritic fEPSP was $49 \pm 4 \mu \mathrm{A}(N=14)$, and the intensity used for the LTP-inducing stimulation train was $50 \pm 5 \mu \mathrm{A}(N=14)$.

Seven of 14 rats showed stable responses across experiments that involved all four behavioral states (see Materials and Methods) (Fig. 1 $A$ ). In Figure $1 A$, the tetanus (open arrow) was delivered to activate the basal dendrites of CA1 of a rat during walking, which was accompanied by a theta rhythm of $7-8 \mathrm{~Hz}$ theta. After the tetanus, responses at both surface and deep electrodes showed a robust increase (compare $30 \mathrm{~min}$ vs control $0 \mathrm{~min}$ ), and the increase persisted to $120 \mathrm{~min}$ and the next day (Fig. $1 A$, right). In the next experiment, 1 week later, the tetanus was delivered in the same rat during REM. A regular theta rhythm was also observed 
during REM, and the rat was in a sleep posture (Fig. $1 B$, left), and it remained in REM for $>10 \mathrm{sec}$ after the tetanus. The enhancement of fEPSPs was also found at both R1 and R2 electrodes after the tetanus in REM (Fig. $1 B$, right), but it was not as large as that after the tetanus given during walking (Fig. $1 A$, right). In the third experiment using this rat, a tetanus was delivered during SWS when slow waves were found in the hippocampal EEG and the rat was in a sleep posture (Fig. $1 C$, left). Enhancement of the fEPSPs was found after a tetanus given in SWS, and its magnitude was comparable to that after a tetanus given in REM (Fig. $1 C$, right). In the last experiment shown, the tetanus was delivered during IMM, during which the hippocampal EEG was mainly irregular but occasionally mixed with a theta rhythm (Fig. 1D). fEPSP enhancement induced by a tetanus given during IMM (Fig. $1 D$, right) was comparable or larger than that induced during the sleep states (REM or SWS), but smaller than that induced during walking (Fig. $1 A$ ).

Similar magnitudes of fEPSP enhancement (LTP) were found at electrode R2, the electrode near the alvear surface, as compared with electrode $\mathrm{R} 1$ at the stratum radiatum (Fig. 1). Subsequent analyses will be presented only for the radiatum electrode responses, which had a larger signal (Materials and Methods). LTP was not restricted to one stimulus intensity, as shown by the inputoutput curves of the fEPSPs (Fig. 2A). For various stimulus intensities, LTP was also larger when the tetanus was delivered during walking as compared with any other state (Fig. 2A).

Group data in seven rats confirmed the results described for the rat illustrated in Figure 1. Experiments on the seven rats did not cover all the 24 permutations of the sequences of four behavioral states. However, in this group of rats, the order of experiments was quasi-random, and the average sequence order of each behavioral state was 2.1-2.7 (1 was assigned to the first experiment of the sequence, and 4 to the last experiment), and this sequence order did not differ among the four behavioral states. In the group, the mean LTP induced during walking was larger than the mean LTP induced during any other behavioral state, including IMM, SWS, or REM (Fig. 2 B). Repeated measures block ANOVA showed a significant behavioral-state effect $\left(F_{(3,18)}=5.05 ; p<0.02\right)$, a significant time effect $\left(F_{(9,54)}=27.9 ; p<\right.$ $0.0001)$, but no significant state $\times$ time interaction $\left(F_{(27,162)}=1.28\right.$; $p>0.17)$. The LTPs induced during IMM, SWS, and REM were not different among each other (Fig. $2 B$ ).

Additional experiments were performed for comparing LTP induced in two different behavioral states (see Materials and Methods). Ten rats were given tetanus during walking and IMM, in a randomized order. LTP induced during walking was larger than that induced during IMM for these rats; repeated measures ANOVA revealed a significant main effect $\left(F_{(1,9)}=13.3 ; p<\right.$
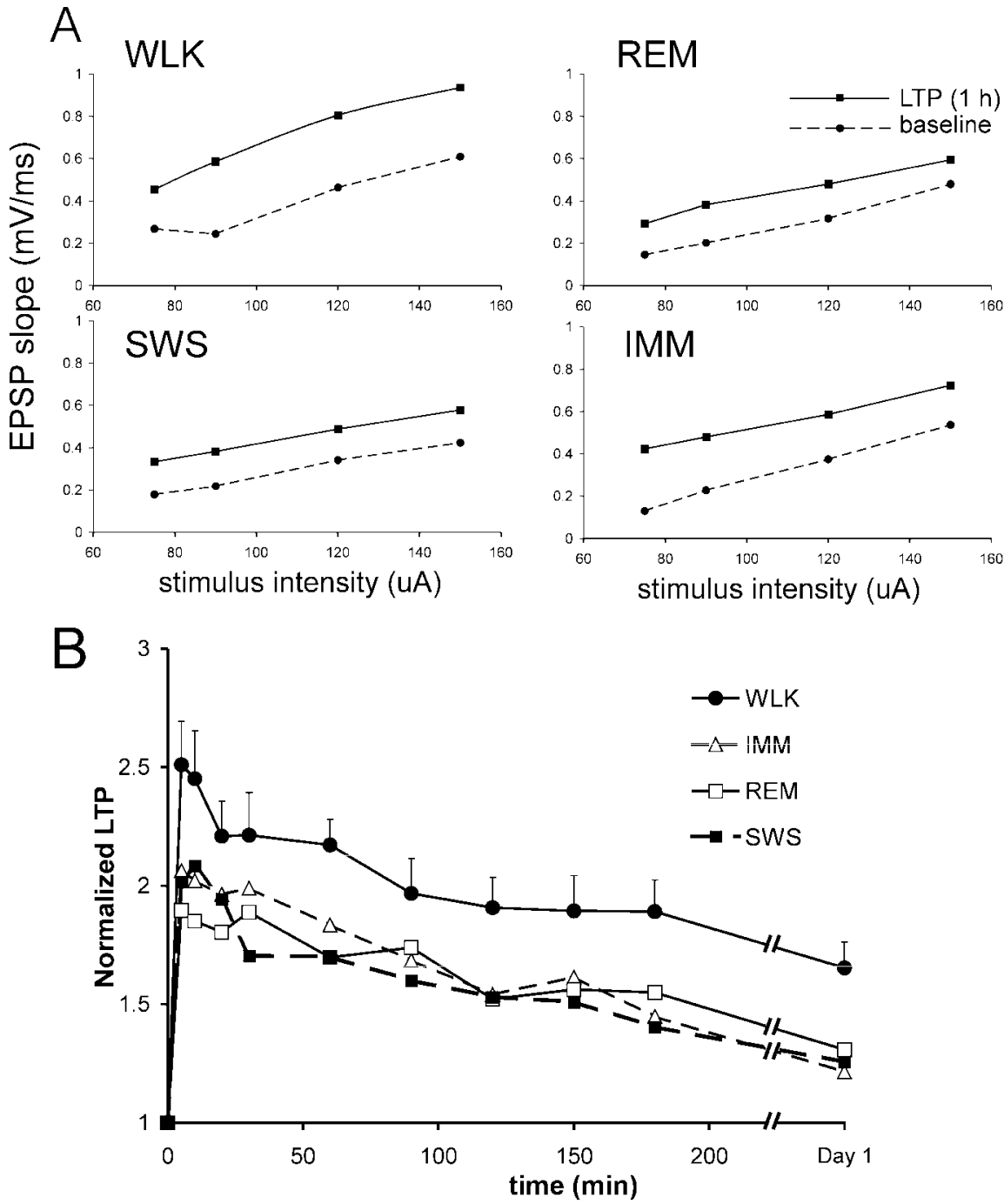

Figure 2. Behavior-dependent basal-dendritic LTP is shown for different stimulus intensities $(A)$ and a group of rats $(B)$. $A$, Input - output curves show that LTP was found at various stimulus intensities of the test pulse, for experiments in which LTP was induced during one of the four behavioral states: WLK, REM, SWS, and IMM. Same experiments as shown in Figure 1 (rat 913). The were stable across experiments, and the tetanus was given, in a pseudorandom order, in all four behavioral states. Baseline slope of the fEPSP was normalized to one in each rat. For clarity, the error bars (SEM) are only shown for LTP induced during walking. The magnitude of the SEM is generally similar for LTP induced during other behavioral states.

0.01). When comparing the LTP induced during SWS with that induced during REM or IMM, there was no main (group) effect (both comparisons, $F_{(1,7)}<0.5 ; p>0.5$ ) or group $\times$ time interactions. In particular, LTP induced during walking was larger than that induced during $\operatorname{REM}\left(F_{(1,7)}=11.8 ; p<0.02\right)$. Both walking and REM states were accompanied by a regular hippocampal theta rhythm. However, analysis indicated that the average theta frequency immediately preceding the tetanus during walking was $7.8 \pm 0.2 \mathrm{~Hz}(N=8)$, significantly higher than the frequency of $6.8 \pm 0.2 \mathrm{~Hz}(N=8)$ immediately preceding the tetanus during REM.

\section{Effect of muscarinic cholinergic blockade on LTP during waking states}

Because the release of acetylcholine increased during walking as compared with IMM (Dudar et al., 1979), the increased cholinergic activity during walking may be responsible for the enhance- 

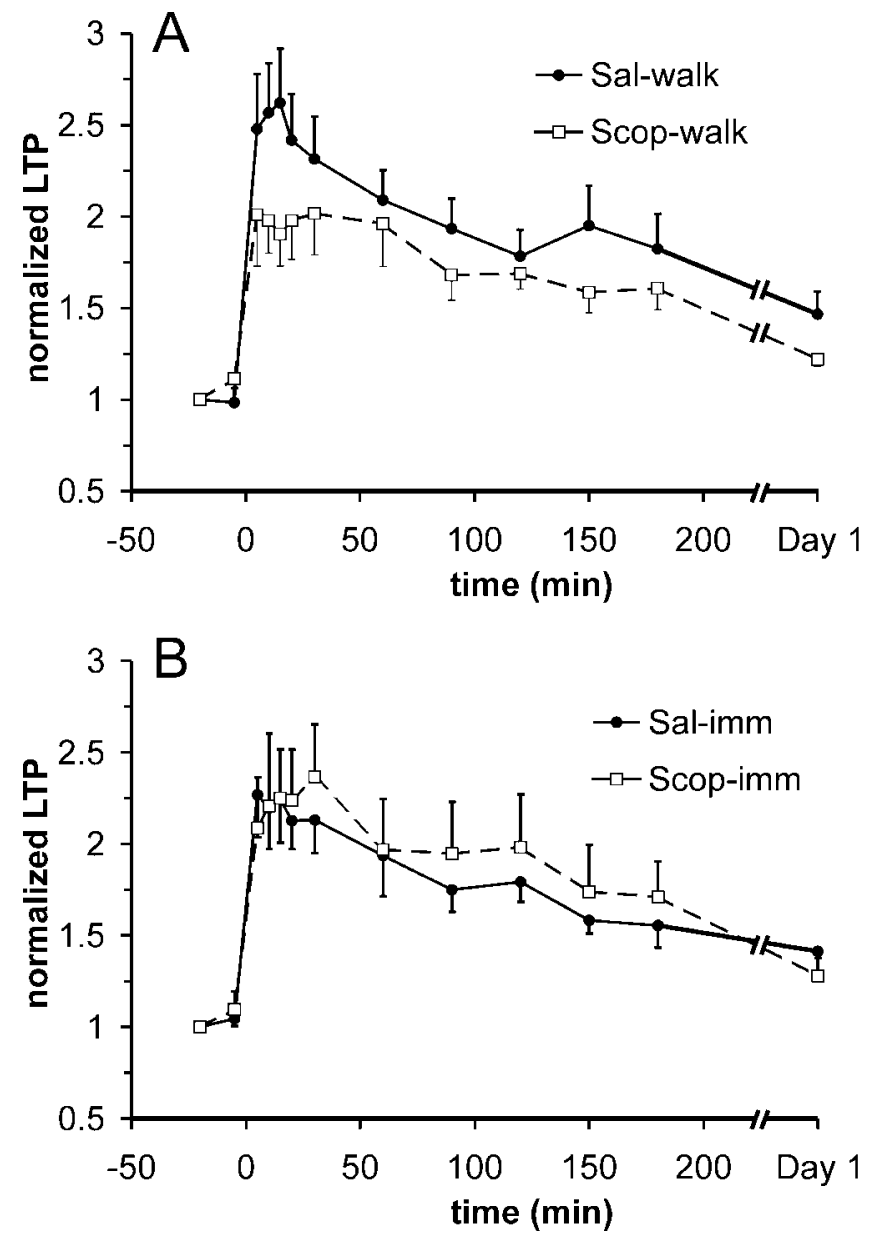

Figure 3. Basal-dendritic LTP during walking but not immobility in normal rats was suppressed by muscarinic cholinergic antagonist scopolamine $(5 \mathrm{mg} / \mathrm{kg}$, i.p.). A, Average normalized LTP, with SEM (one-way error bars), induced by a tetanus given during walking with saline intraperitoneal injection (Sal-walk) is significantly different from the LTP induced during walking with pretreatment of scopolamine (Scop-walk); see Results for details. B, Average normalized LTP (mean and SEM) induced during awake-immobility with saline pretreatment (Sal$i m m$ ) compared with that induced during immobility with scopolamine pretreatment (Scopimm). LTP induced during IMM was not significantly affected by scopolamine.

ment of LTP. To block the effects of muscarinic cholinergic inputs, eight rats were pretreated with scopolamine hydrochloride ( $5 \mathrm{mg} / \mathrm{kg}$, i.p.) or saline before a tetanus was given during walking. Average LTP was smaller in rats in which the tetanus was delivered during walking after scopolamine, as compared with when the tetanus was delivered during walking after saline (intraperitoneal) injection (Fig. $3 A$ ) or without injection (data not shown). Repeated measures ANOVA of the LTP revealed a marginally insignificant scopolamine treatment effect $\left(F_{(1,7)}=4.39\right.$; $p=0.074)$, but significant time $\left(F_{(10,70)}=14.9 ; p<0.0001\right)$ and treatment $\times$ time $\left(F_{(10,70)}=2.92 ; p=0.0039\right)$ effects. Post hoc Newman-Keuls showed that LTP, when tetanized during walking, was significantly smaller after scopolamine treatment than after saline treatment at times 5-30 $\mathrm{min}, 150 \mathrm{~min}$, and $1 \mathrm{~d}$ after tetanus. In six rats given scopolamine or saline before a tetanus during IMM, there was little difference in the LTP induced during scopolamine immobility and saline immobility (Fig. 3B). Repeated measures ANOVA revealed that there was no significant treatment $\left(F_{(1,5)}=1.77 ; p>0.2\right)$ and treatment $\times$ time $\left(F_{(10,50)}\right.$ $=1.17 ; p>0.3)$ effects.
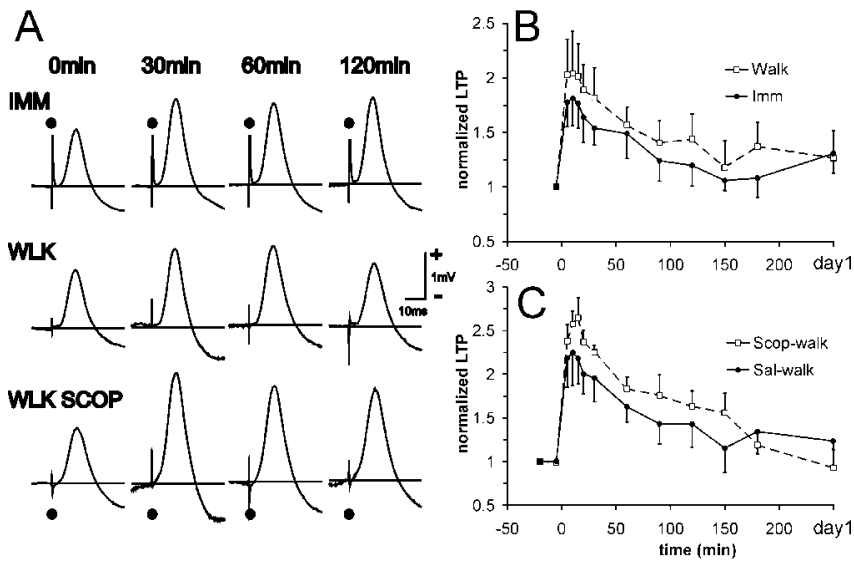

Figure 4. Rats injected with lgG192-SAP did not show LTP that was altered by behavioral states or by scopolamine. A, Representative rat (906) with SAP lesion showing average evoked response (fEPSPs) at CA1 stratum radiatum after basal-dendritic association fiber stimulation, during baseline ( $0 \mathrm{~min})$, and at 30,60, and $120 \mathrm{~min}$ after the tetanus. Three different experiments from the same rat are shown, with the tetanus delivered during IMM, WLK, and WLK SCOP. To facilitate comparison, the responses were scaled to make the peak amplitudes of the baseline response appear identical (without scaling, peak baseline responses were within $24 \%$ of each other). Calibration of $1 \mathrm{mV}$ is within $\pm 15 \%$ error, but the calibration is the same for each experiment (row). There was no enhancement of LTP when tetanized during walk compared with IMM, and scopolamine enhanced instead of depressed LTP during walking in this rat. $B$, Normalized basal-dendritic LTP group data, with tetanus given during walking (walk) or during awake-immobility (Imm) (mean and SEM of 5 rats). C, Normalized basal-dendritic LTP group data, comparing experiment with tetanus given during walking with scopolamine (Scop-walk) or saline pretreatment (Sal-wal) (average and SEM of 4 rats). The group differences shown in $B$ and $C$ are not statistically significant.

\section{LTP in IgG192-saporin lesion and sham-lesion rats}

Five rats with cholinotoxin IgG192-SAP injected into the medial septum were tetanized for LTP during different behavioral states. The group of SAP-lesion rats showed basal-dendritic LTP induced during either walking or IMM. However, distinct from normal rats, there was no significant difference in the LTP induced during IMM and that induced during walking (Fig. 4A, $B$ ) $\left(F_{(1,4)}=1.46 ; p>0.29\right)$, and there was no significant group $\times$ time interaction. The representative rat illustrated (Fig. 4A) showed slightly larger fEPSPs after a tetanus delivered during IMM than during walking. An even more notable effect was an enhancement of fEPSPs (LTP) when scopolamine was injected before the tetanus during walking (Fig. $4 A$, WLK SCOP) as compared with after a tetanus during undrugged walking (Fig. $4 \mathrm{~A}$, WLK). As a group, the SAP-lesion rats were distinct from normal rats in that LTP during walking was not significantly affected by scopolamine pretreatment (Fig. $4 C)\left(F_{(1,3)}=2.74 ; p>0.19\right.$, with no significant group $\times$ time interaction). As expected, ChATimmunoreactive cells in the medial septum were greatly diminished in the SAP-lesion rats (Fig. 5A), in both the medial septum and vertical limb of the diagonal band, as compared with normal or sham-lesion rats (Fig. $5 B$ ). The number of parvalbuminpositive cells (presumably GABAergic neurons) in the medial septum and diagonal band was not significantly altered in SAPlesion rats (data not shown). There was also a pronounced loss of AchE staining in the hippocampus in SAP-lesion rats (Fig. 5C) as compared with sham-lesion rats (Fig. 5D). Hippocampal theta rhythm in SAP-lesion rats was decreased in amplitude, but not in frequency, as compared with sham-lesion rats (data not shown), as reported by Zheng and Khanna (2001).

In each of the four sham-lesion rats, similar to normal control rats, the basal-dendritic LTP elicited during walking was larger 
than the LTP induced during IMM. Repeated measures ANOVA showed no group effect $\left(F_{(1,3)}=4.21 ; p=0.13\right)$ but a significant group $\times$ time interaction $\left(F_{(10,30)}=2.6 ; p=0.02\right)$. Post hoc Newman-Keuls revealed that LTP induced during walk and IMM states were significantly different $(p<0.05)$ at times $5-150$ min after tetanus. In addition, LTP induced during walking and pretreated with scopolamine was smaller than LTP of the same rats induced during walking without scopolamine; repeated measures ANOVA yielded a significant drug effect $\left(F_{(1,3)}=\right.$ $35.2 ; p<0.01)$ but no drug $\times$ time interactions. In summary, basal-dendritic LTP of sham-lesion rats resembled LTP of normal control rats.

\section{Discussion}

Basal-dendritic LTP is dependent on the behavioral state during induction

We demonstrated original results that the basal-dendritic LTP in CA1 was modulated by behavioral states. We showed that LTP was largest when a high-frequency tetanus was delivered during walking as compared with IMM, SWS, or REM, and that the LTP enhancement was mediated by septohippocampal cholinergic inputs. Systemic muscarinic cholinergic blockade abolished the LTP enhancement during walking as compared with immobility. Selective cholinotoxic (IgG192-saporin) lesion of the medial septum also abolished the LTP enhancement when LTP induction occurred during walking, as compared with immobility.

The larger LTP induced during walking as compared with that induced during any other behavioral state may be interpreted as an enhanced cholinergic input to the hippocampus during walking than any other state. The larger acetylcholine release during walking than immobility (Dudar et al., 1979) has been demonstrated, and higher cholinergic function was inferred during walking as compared with IMM or SWS (Leung, 1980; Leung and Vanderwolf, 1980; Leung, 1998).

Because both walking and REM are associated with high cholinergic inputs (Vanderwolf, 1988), the larger LTP induced during walking than that induced during REM may not be expected if cholinergic inputs were the sole factor. One explanation is that theta frequency could account for the difference in LTP during walking as compared with REM. The LTP induction during REM occurred at an average theta frequency of $6.8 \mathrm{~Hz}(5-7.5 \mathrm{~Hz}$ range), whereas LTP induction during walking occurred at mean theta frequency of $7.8 \mathrm{~Hz}$. REM has been classified into tonic and phasic phases (Jouvet, 1967; Vanderwolf, 1988), and at least in some rats, the LTP was induced during tonic REM during which vibrissae twitching and limb jerking were absent. Higher acetylcholine release was shown to accompany a higher theta frequency $(4-7 \mathrm{~Hz})$ in the urethane-anesthetized rat (Keita et al., 2000). Similarly, cholinergic modulation of the hippocampus increased with the frequency of the movement-associated (type I) theta rhythm in the behaving rat (Leung and Vanderwolf, 1980). Thus, a larger LTP during walking than during REM may still be consistent with cholinergic modulation of LTP, but the lack of LTP enhancement during REM as compared with SWS requires a different explanation. One possibility is that the lack of seroto- nergic and noradrenergic inputs during REM may prevent the cholinergic enhancement of hippocampal LTP. During REM, the forebrain including the hippocampus is deprived of serotonergic and noradrenergic inputs, because raphe and locus coeruleus neurons stopped firing completely (Steriade and McCarley, 1990). It is known that $\beta$-adrenergic receptor activation acted synergistically with cholinergic activation in enhancing LTP in CA1 in vitro (Watabe et al., 2000), and depletion of serotonin (5-HT) and norepinephrine reduced LTP in the DG of anesthetized rats (Bliss et al., 1983). However, different results were found in behaving rats; norepinephrine depletion enhanced LTP of the fEPSPs in the DG (Robinson and Racine, 1985), and 5-HT3 receptor blockade enhanced LTP in CA1 (Staubli and Xu, 1995). Other than cholinergic inputs, the high-frequency theta rhythm in behaving rats is associated with an atropine-resistant noncholinergic inputs, suggested to be mediated by 5-HT, GABA, or glutamate (Vanderwolf, 1988; Leung, 1998; Wu et al., 2000; Buzsaki, 2002). The participation of these behaviorally associated noncholinergic inputs in LTP remains to be studied.

\section{Cholinergic modulation of LTP}

We showed that lesion of the septohippocampal cholinergic neurons and muscarinic antagonists blocked the behavioral enhancement of LTP, but they did not abolish LTP per se. Systemic injection of scopolamine is expected to block muscarinic receptors in all parts of the brain, but injection of IgG192-saporin is selective for cholinergic septohippocampal neurons with p75 receptors (Wiley, 1992; Lee et al., 1994; Zheng and Khanna, 2001). Thus, in combination, both systemic scopolamine and SAP lesion support the hypothesis that cholinergic inputs impinging on muscarinic receptors in the hippocampus are responsible for the larger LTP induced during walking than during immobility. In previous studies, muscarinic blockade or lesion of cholinergic neurons was reported not to affect hippocampal LTP (FeaseyTruger et al., 1992; Jouvenceau et al., 1996; Kikusui et al., 2000), but behaviorally dependent LTP was not studied.

The mechanisms by which acetylcholine may enhance basaldendritic LTP are not known. Various actions of muscarinic cho- 
linergic receptors, including blockade of $\mathrm{K}^{+}$-mediated conductances (Krnjevic, 1993), disinhibition of CA1 pyramidal cells (Krnjevic et al., 1988), and enhancement of NMDA receptor currents (Auerbach and Segal, 1996), may provide the cholinergic enhancement of LTP. Excitatory synapses innervating the basal dendrites rather than those innervating the apical dendrites of CA1 pyramidal cells appears to be more favorably inclined toward cholinergic enhancement of LTP. Apical-dendritic EPSPs and population spikes were suppressed by a muscarinic cholinergic input during walking (Leung, 1980), whereas basaldendritic EPSPs were not significantly altered during walking as compared with IMM (Leung, 1980, 1998), and the basaldendritic population spike was enhanced during walking as compared with IMM (Buzsaki et al., 1981). It is known that muscarinic acetylcholine receptors mediate presynaptic inhibition of glutamatergic synapses on the apical dendrites of CA1 pyramidal cells (Valentino and Dingledine, 1981). Behavioral modulation of apical-dendritic LTP has not been systematically studied, but preliminary data (data not shown) suggest that it was not readily detectable.

Several studies have shown a cholinergic enhancement of hippocampal LTP in vitro, mainly by using cholinergic agonists or AchE inhibitors (Hirotsu et al., 1989; Blitzer et al., 1990). Increase of LTP in CA1 by septal stimulation was shown to be dependent on muscarinic cholinergic receptors (Galey et al., 1994; Markevich et al., 1997), and LTP in the DG was facilitated by muscarinic cholinergic activation (Abe et al., 1994). Cholinesterase inhibitors were reported to extend the duration of LTP in the DG of old animals (Barnes et al., 2000). In distinction to the previous studies, the enhancement of LTP in the present study depends on the higher physiological release of acetylcholine during walking than immobility (Dudar et al., 1979) rather than by pharmacological manipulation or electrical stimulation.

Behavioral modulation may serve an important role in hippocampal function (Leung, 1998). The long-term alteration of synaptic weights in LTP may underlie memory in an animal (Morris and Frey, 1997; Hasselmo, 1999), although whether saturation or blockade of LTP at a particular synapse (entorhinal cortex to DG) blocks spatial memory has been disputed (Morris et al., 1986; Barnes et al., 1994; Saucier and Cain, 1995; Moser et al., 1998). However, memory function in the hippocampus likely resides in multisynaptic and parallel entorhinal-hippocampal circuits (Amaral and Witter, 1989; Canning et al., 2000), and the recurrent $\mathrm{CA} 3$ or CA3 to CA1 connections may be necessary for recall when spatial cues were incomplete (Brun et al., 2002; Nakazawa et al., 2002). Hippocampal LTP is proposed to underlie the formation of the firing field of place cells (Muller et al., 1996), which also required active exploration of the environment by a rat (Foster et al., 1989). Active exploration will be accompanied by a large cholinergic input to the hippocampus, and muscarinic cholinergic blockade was reported to disrupt place fields (Brazhnik et al., 2003). Among the synaptic connections of CA3 to the dendrites of CA1, LTP at the basal dendrites is more robust than that at the apical dendrites (Kaibara and Leung, 1993; Arai et al., 1994; Leung and Shen, 1995). Thus, the cumulative evidence suggests that behavioral modulation of basal-dendritic LTP by cholinergic inputs is of important functional significance.

\section{References}

Abe K, Nakata A, Mizutani A, Saito H (1994) Facilitatory but nonessential role of the muscarinic cholinergic system in the generation of long-term potentiation of population spikes in the dentate gyrus in vivo. Neuropharmacology 33:847-852.
Amaral DG, Witter MP (1989) The three-dimensional organization of the hippocampal formation: a review of anatomical data. Neuroscience 31:571-591.

Arai A, Black J, Lynch G (1994) Origins of the variations in long-term potentiation between synapses in the basal versus apical dendrites of hippocampal neurons. Hippocampus 4:1-9.

Auerbach JM, Segal M (1996) Muscarinic receptors mediating depression and long-term potentiation in rat hippocampus. J Physiol (Lond) 492:479-493.

Barnes CA, Jung MW, McNaughton BL, Korol DL, Andreasson K, Worley PF (1994) LTP saturation and spatial learning disruption: effects of task variables and saturation levels. J Neurosci 14:5793-5806.

Barnes CA, Meltzer J, Houston F, Orr G, McGann K, Wenk GL (2000) Chronic treatment of old rats with donepezil or galantamine: effects on memory, hippocampal plasticity and nicotinic receptors. Neuroscience 99:17-23.

Bartus RT (2000) On neurodegenerative diseases, models, and treatment strategies: lessons learned and lessons forgotten a generation following the cholinergic hypothesis. Exp Neurol 163:495-529.

Bliss TVP, Collingridge GL (1993) A synaptic model of memory: Long-term potentiation in the hippocampus. Nature 361:31-39.

Bliss TVP, Gardner-Medwin AR (1973) Long-lasting potentiation of synaptic transmission in the dentate area of the unanaesthetized rabbit following stimulation of the perforant path. J Physiol (Lond) 232:357-374.

Bliss TVP, Lomo T (1973) Long-lasting potentiation of synaptic transmission in the dentate area of the anaesthetized rabbit following stimulation of the perforant path. J Physiol (Lond) 232:331-356.

Bliss TVP, Goddard GV, Riives M (1983) Reduction of long-term potentiation in the dentate gyrus of the rat following selective depletion of monoamines. J Physiol (Lond) 334:475-491.

Blitzer RD, Gil O, Landau EM (1990) Cholinergic stimulation enhances long-term potentiation in the CA1 region of rat hippocampus. Neurosci Lett 119:207-210.

Boyd TE, Trepel C, Racine RJ (2000) Cholinergic modulation of neocortical long-term potentiation in the awake, freely moving rat. Brain Res 881:28-36.

Bramham CR, Srebro B (1989) Synaptic plasticity in the hippocampus is modulated by behavioural state. Brain Res 493:74-86.

Brun VH, Otnass MK, Molden S, Steffenach HA, Witter MP, Moser MB, Moser EI (2002) Place cells and place recognition maintained by direct entorhinal- hippocampal circuitry. Science 296:2243-2246.

Brazhnik ES, Muller RU, Fox SE (2003) Muscarinic blockade slows and degrades the location-specific firing of hippocampal pyramidal cells. J Neurosci 23:611-621.

Buzsaki G (1989) Two-stage model of memory trace formation: a role for "noisy" brain states. Neuroscience 31:551-570.

Buzsaki G (2002) Theta oscillations in the hippocampus. Neuron 33:325-340.

Buzsaki G, Grastyan E, Czopf J, Kellenyi L, Prohaska O (1981) Changes in neuronal transmission in the rat hippocampus during behavior. Brain Res 225:235-247.

Canning KJ, Wu K, Peloquin P, Kloosterman F, Leung LS (2000) Physiology of the entorhinal and perirhinal projections to the hippocampus studied by current source density analysis. Ann NY Acad Sci 911:55-72.

Decker MW, McGaugh JL (1991) The role of interactions between the cholinergic system and other neuromodulatory systems in learning and memory. Synapse 7:151-168.

Deutsch JA (1991) The cholinergic synapse and the site of memory. Science 174:788-794.

Dudar JD, Whishaw IQ, Szerb JC (1979) Release of acetylcholine from the hippocampus of freely moving rats during sensory stimulation and running. Neuropharmacology 18:673-678.

Feasey-Truger KJ, Li BH, ten Bruggencate G (1992) Lesions of the medial septum which produce deficits in working/spatial memory do not impair long-term potentiation in the $\mathrm{CA} 3$ region of the rat hippocampus in vivo. Brain Res 591:296-304.

Foster TC, Castro CA, McNaughton BL (1989) Spatial selectivity of rat hippocampal neurons: dependence on preparedness for movement. Science 25:1580-1582.

Galey D, Destrade C, Jaffard R (1994) Relationships between septohippocampal cholinergic activation and the improvement of long-term 
retention produced by medial septal electrical stimulation in two inbred strains of mice. Behav Brain Res 60:183-189.

Gallagher M, Nicolle MM (1993) Animal models of normal aging: relationship between cognitive decline and markers in hippocampal circuitry. Behav Brain Res 57:155-162.

Hasselmo ME (1999) Neuromodulation: acetylcholine and memory consolidation. Trends Cogn Sci 3:351-359.

Hasselmo ME, Barkai E (1995) Cholinergic modulation of activity-dependent synaptic plasticity in the piriform cortex and associative memory function in a network biophysical simulation. J Neurosci 15:6592-6604.

Hirotsu I, Hori N, Katsuda N, Ishihara T (1989) Effect of anticholinergic drug on long-term potentiation in rat hippocampal slices. Brain Res 482:194-197.

Holscher C, Anwyl R, Rowan MJ (1997) Stimulation on the positive phase of hippocampal theta rhythm induces long-term potentiation which can be depotentiated by stimulation on the negative phase in area CA1 in vivo. J Neurosci 17:6470-6477.

Jones Leonard B, McNaughton BL, Barnes CA (1987) Suppression of hippocampal synaptic plasticity during slow-wave sleep. Brain Res 425:174-177.

Jouvenceau A, Billard JM, Lamour Y, Dutar P (1996) Persistence of CA1 hippocampal LTP after selective cholinergic denervation. NeuroReport 7:948-952.

Jouvet M (1967) Neurophysiology of the states of sleep. Physiol Rev $47: 117-177$.

Kaibara T, Leung LS (1993) Basal versus apical dendritic long-term potentiation of commissural afferents to hippocampal CA1: a current-source density study. J Neurosci 13:2391-2404.

Kasa P, Rakonczay Z, Gulya K (1997) The cholinergic system in Alzheimer's disease. Prog Neurobiol 52:511-535.

Keita MS, Frankel-Kohn L, Bertrand N, Lecanu L, Monmaur P (2000) Acetylcholine release in the hippocampus of the urethane anaesthetised rat positively correlates with both peak theta frequency and relative power in the theta band. Brain Res 887:323-334

Kikusui T, Aoyagi A, Kaneko T (2000) Spatial working memory is independent of hippocampal CA1 long-term potentiation in rats. Behav Neurosci 114:700-706.

Krnjevic K (1993) Central cholinergic mechanisms and function. Prog Brain Res 98:285-292.

Krnjevic K, Ropert N, Casullo J (1988) Septohippocampal disinhibition. Brain Res 438:182-192.

Lee MG, Chrobak JJ, Sik A, Wiley RG, Buzsaki G (1994) Hippocampal theta activity following selective lesion of the septal cholinergic system. Neuroscience 62:1033-1047.

Leung LS (1980) Behaviour dependent evoked potentials in the hippocampal CA1 region of the rat. I. Correlation with behaviour and EEG. Brain Res 198:95-117.

Leung LS (1987) Hippocampal electrical activity following local tetanization. I. Afterdischarges. Brain Res 419:173-187.

Leung LS (1998) Generation of theta and gamma rhythms in the hippocampus. Neurosci Biobehav Rev 22:275-290.

Leung LS, Shen B (1995) Long-term potentiation at the apical and basal dendritic synapses of CA1 after local stimulation in behaving rats. J Neurophysiol 73:1938-1946.

Leung LS, Shen B (1999) N-methyl-D-aspartate receptor antagonists are less effective in blocking long-term potentiation at apical than basal dendrites in hippocampal CA1 of awake rats. Hippocampus 9:617-630.

Leung LS, Vanderwolf CH (1980) Behav-dependent evoked potentials in the hippocampal CA1 region of the rat. II. Effect of eserine, atropine, ether and pentobarbital. Brain Res 198:119-133.

Leung LS, Shen B, Kaibara T (1992) Long-term potentiation induced by patterned stimulation of the commissural pathway to hippocampal CA1 region in freely moving rats. Neuroscience 48:63-74.

Louie K, Wilson MA (2001) Temporally structured replay of awake hippocampal ensemble activity during rapid eye movement sleep. Neuron 29:145-156.

Markevich V, Scorsa AM, Dawe GS, Stephenson JD (1997) Cholinergic facilitation and inhibition of long-term potentiation of CA1 in the urethane-anaesthetized rats. Brain Res 754:95-102.

Morris GRM, Frey U (1997) Hippocampal synaptic plasticity: role in spatial learning or the automatic recording of attended experience? Philos Trans R Soc Lond B Biol Sci 352:1489-1503.

Morris RGM, Anderson E, Lynch GS, Baudry M (1986) Selective impairment of learning and blockade of long-term potentiation by an $N$-methylD-aspartate receptor antagonist, AP5. Nature 319:774-776.

Moser EI, Krobert KA, Moser M, Morris RG (1998) Impaired spatial learning after saturation of long-term potentiation. Science 281:2038-2042.

Muller RU, Stead M, Pach J (1996) The hippocampus as a cognitive graph. J Gen Physiol 107:663-694.

Nakazawa K, Quirk MC, Chitwood RA, Watanabe M, Yeckel MF, Sun LD, Kato A, Carr CA, Johnston D, Wilson MA, Tonegawa S (2002) Requirement for hippocampal CA3 NMDA receptors in associative memory recall. Science 297:211-218.

Pavlides C, Winson J (1989) Influences of hippocampal place cell firing in the awake state on the activity of these cells during subsequent sleep episodes. J Neurosci 9:2907-2918.

Pavlides C, Greenstein YJ, Grudman M, Winson J (1988) Brain Res 439:383-387.

Paxinos G, Watson C (1986) The rat brain in stereotaxic coordinates, Ed 2. San Diego: Academic.

Robinson GB, Racine RJ (1985) Long-term potentiation in the dentate gyrus: effects of noradrenaline depletion in the awake rat. Brain Res 325:71-78.

Saucier D, Cain DP (1995) Spatial learning without NMDA receptordependent long-term potentiation. Nature 378:186-189.

Sirvio J (1999) Strategies that support declining cholinergic neurotransmission in Alzheimer's disease patients. Gerontology [Suppl 1] 45:3-14.

Smith C (1996) Sleep states, memory processes and synaptic plasticity. Behav Brain Res 78:49-56.

Staubli U, Xu FB (1995) Effects of 5-HT3 receptor antagonism on hippocampal theta rhythm, memory, and LTP induction in the freely moving rat. J Neurosci 15:2445-2452.

Steriade M, McCarley RW (1990) Brainstem control of wakefulness and sleep. New York: Plenum.

Vanderwolf CH (1988) Cerebral activity and behavior: control by cholinergic and serotonergic systems. Int Rev Neurobiol 30:225-340.

Valentino R, Dingledine R (1981) Presynaptic inhibitory effect of acetylcholine in the hippocampus. J Neurosci 1:784-792.

Watabe AM, Zaki PA, O'Dell TJ (2000) Coactivation of beta-adrenergic and cholinergic receptors enhances the induction of long-term potentiation and synergistically activates mitogen-activated protein kinase in the hippocampal CA1 region. J Neurosci 20:5924-5931.

Wiley RG (1992) Neural lesioning with ribosome-inactivating proteins: suicide transport and immunolesioning. Trends Neurosci 15:285-290.

Wilson MA, McNaughton BL (1994) Reactivation of hippocampal ensemble memories during sleep. Science 265:676-679.

Wu M, Shanabrough M, Leranth C, Alreja M (2000) Cholinergic excitation of septohippocampal GABA but not cholinergic neurons: implications for learning and memory. J Neurosci 20:3900-3908.

Zheng F, Khanna S (2001) Selective destruction of medial septal cholinergic neurons attenuates pyramidal cell suppression, but not excitation in dorsal hippocampus field CA1 induced by subcutaneous injection of formalin. Neuroscience 103:985-998. 